\title{
Determinação de selênio em leite bovino in natura provenientes do Ceará - Brasil
}

\author{
Determination of selenium in bovine millk in natura from Ceará - Brazil \\ Determinación de selenio en leche bovina in natura de Ceará - Brasil
}

Recebido: 12/05/2021 | Revisado: 18/05/2021 | Aceito: 21/05/2021 | Publicado: 07/06/2021

\author{
Renata Carmo de Assis \\ ORCID: https://orcid.org/0000-0003-2258-3844 \\ Universidade Estadual do Ceará, Brasil \\ E-mail: renata.carmo.assis@gmail.com \\ Maria Dinara de Araújo Nogueira \\ ORCID: https://orcid.org/0000-0001-7843-9089 \\ Universidade Estadual do Ceará, Brasil \\ E-mail: dinara.araujo@alumo.uece.br \\ Alexandre Danton Viana Pinheiro \\ ORCID: https://orcid.org/0000-0001-9180-5514 \\ Universidade Estadual do Ceará, Brasil \\ E-mail: alexandredanton@hotmail.com \\ Ruth Silva Galdino \\ ORCID: https://orcid.org/0000-0003-4936-8888 \\ Universidade Estadual do Ceará, Brasil \\ E-mail: ruth.galdino@aluno.uece.br \\ Kaluce Gonçalves de Sousa Almondes \\ ORCID: https://orcid.org/0000-0003-2694-1629 \\ Universidade Estadual do Ceará, Brasil \\ E-mail: kaluce.almondes@uece.br \\ Carla Soraya Costa Maia \\ ORCID: https://orcid.org/0000-0003-1535-6686 \\ Universidade Estadual do Ceará, Brasil \\ E-mail: carla.maia@uece.br
}

\begin{abstract}
Resumo
O selênio é essencial para a saúde humana. Dentre os alimentos considerados fonte de selênio, estão as castanhas brasileiras, carnes, ovos, leites e outros, variando conforme a área geográfica de plantio ou criação do animal. Dados de Se em alimentos cultivados, industrializados e comercializados no Brasil são ausentes em tabelas de composição de alimentos brasileiras. O objetivo do estudo foi determinar a concentração de selênio em leite bovino in natura produzido no Ceará, região Nordeste do Brasil. Trata-se de uma pesquisa experimental, onde as amostras foram coletadas em municípios das principais bacias leiteiras do Ceará. As concentrações de selênio foram determinadas por Espectrofotometria de Absorção Atômica por Geração de Hidretos acoplado a cela de quartzo. Os teores de umidade, cinzas e proteína foram analisados segundo a metodologia da Association of Official Analytical Chemists (1980), o teor de lipídios por método de Soxhlet e a quantidade de carboidratos foi calculada por diferença. A média das concentrações de selênio, umidade, cinza, proteína, lipídio e carboidrato do leite bovino in natura foi de 2.9 $\mu \mathrm{g} / 100 \mathrm{~mL}, 89.0 \mathrm{mg} / 100 \mathrm{~mL}, 0.6 \mathrm{mg} / 100 \mathrm{~mL}, 2.9 \mathrm{mg} / 100 \mathrm{~mL}, 3.6 \mathrm{mg} / 100 \mathrm{~mL}$ e $4.0 \mathrm{mg} / 100 \mathrm{~mL}$, respectivamente. A concentração de selênio no leite bovino in natura variou de $0.1-9.0 \mu \mathrm{g} / 100 \mathrm{ml}$, sendo em algumas localidades superior em relação a outras localidades do Brasil e do mundo, podendo contribuir para o consumo deste mineral pela população do estado.
\end{abstract}

Palavras-chave: Selênio; Composição de alimentos; Solo; Micronutriente.

\begin{abstract}
Selenium is essential to human health. Among the foods considered to be a source of selenium are Brazilian nuts, meat, eggs, milk and others, varying according to the geographical area where they are grown or raised. Data on Se in foods grown, processed and marketed in Brazil are absent in Brazilian food composition tables. The aim of the study was to determine the selenium concentration in bovine milk in natura produced in Ceará, Northeast region of Brazil. This is an experimental research, where samples were collected in municipalities of the main milk basins of Ceará. The selenium concentrations were determined by Hydride Generation Atomic Absorption Spectrophotometry coupled to a quartz cell. The moisture, ash and protein contents were analyzed according to the Association of Official Analytical Chemists (1980) methodology, the lipid content by Soxhlet method and the amount of carbohydrates was calculated by difference. The mean selenium, moisture, ash, protein, lipid and carbohydrate concentrations of fresh bovine milk were $2.9 \mu \mathrm{g} / 100 \mathrm{~mL}, 89.0 \mathrm{mg} / 100 \mathrm{~mL}, 0.6 \mathrm{mg} / 100 \mathrm{~mL}, 2.9 \mathrm{mg} / 100 \mathrm{~mL}, 3.6 \mathrm{mg} / 100 \mathrm{~mL}$ and $4.0 \mathrm{mg} / 100 \mathrm{~mL}$, respectively. The concentration of selenium in fresh bovine milk ranged from $0.1-9.0 \mu \mathrm{g} / 100 \mathrm{~mL}$, being higher in
\end{abstract}


some locations compared to other locations in Brazil and worldwide, which may contribute to the consumption of this mineral by the population of the state.

Keywords: Selenium; Food composition; Soil; Micronutrient.

\section{Resumen}

El selenio es esencial para la salud humana. Entre los alimentos considerados fuente de selenio se encuentran las nueces brasileñas, la carne, los huevos, la leche y otros, variando según la zona geográfica de siembra o cría de animales. Los datos de Se en alimentos cultivados, procesados y comercializados en Brasil están ausentes en las tablas de composición de alimentos brasileños. El objetivo del estudio fue determinar la concentración de selenio en leche fresca bovina producida en Ceará, región noreste de Brasil. Se trata de una investigación experimental, donde las muestras fueron recolectadas en municipios de las principales cuencas lecheras de Ceará. Las concentraciones de selenio se determinaron mediante espectrofotometría de absorción atómica de hidrocarburos acoplada a la celda de cuarzo. Los contenidos de humedad, cenizas y proteínas se analizaron según la metodología de la Association of Official Analytical Chemists (1980), el contenido de lípidos por el método de Soxhlet y la cantidad de carbohidratos se calculó por diferencia. La concentración promedio de selenio, humedad, cenizas, proteínas, lípidos y carbohidratos en la leche fresca de bovino fue de $2,9 \mu \mathrm{g} / 100 \mathrm{ml}, 89,0 \mathrm{mg} / 100 \mathrm{ml}, 0,6 \mathrm{mg} / 100 \mathrm{ml}, 2,9 \mathrm{mg} / 100 \mathrm{ml}, 3,6 \mathrm{mg} / 100 \mathrm{ml}$ y $4,0 \mathrm{mg} / 100 \mathrm{ml}$, respectivamente. La concentración de selenio en la leche fresca de bovino osciló entre 0.1-9.0 $\mu \mathrm{g} /$ $100 \mathrm{ml}$, siendo en algunos lugares más alta que en otros lugares de Brasil y el mundo, lo que puede contribuir al consumo de este mineral por parte de la población del estado.

Palabras clave: Selenio; Composición de alimentos; Suelo; Micronutrientes.

\section{Introdução}

O selênio (Se) é um elemento essencial para o bom funcionamento dos organismos humanos e animais (Kieliszek \& Błażejak, 2016). Possui um efeito complexo e dinâmico sobre o corpo humano através das selenoproteínas, desempenhando um papel essencial na proteção antioxidante e no metabolismo hormonal Valea e Georgescu (2018).

Alguns estudos têm mostrado alterações corporais de selênio em indivíduos com doenças crônicas degenerativas, como diabetes mellitus tipo 2, hipotireoidismo congênito e câncer Zhang et al. (2019); Khan, Bule, Rahman, Asif, Niaz et al. (2019); Rostami, Fathollahpour, Abdi, Naderi (2018). Também têm sido observado a deficiência de Se em múltiplas perturbações metabólicas, como na hiperglicemia, na hiperlipidemia e na hiperfenilalaninemia e, além disso, a suplementação deste pode contribuir com a melhora de parâmetros de biomarcadores relacionados à aterosclerose, hipercolesterolemia, diabetes mellitus tipo 1 e fenilcetonúria Wang et al. (2017), além de melhorar as defesas antioxidantes em pacientes renais crônicos, a depender da quantidade ingerida Freitas et al. (2020).

A relação existente entre o consumo de Se e risco de doença é complexa, a qual dependente do status de selênio inicial, metabolismo, genótipo e ingestão, sendo estes considerados fatores determinantes para que o indivíduo consiga atingir níveis de absorção satisfatórios para desempenho de suas funções no organismo Tan, Mo, Lau, e Xu (2019).

Acerca das recomendações de ingestão do mineral, a Recommended Dietary Allowance (RDA) para o selênio é de 55 $\mu \mathrm{g} / \mathrm{dia}$, para homens e mulheres adultos e o Upper Limit (UL) é de $400 \mu \mathrm{g} / \mathrm{dia}$. Já, dentre os alimentos considerados fonte de Se, estão as castanhas brasileiras, carnes, ovos, além de leveduras enriquecidas especificamente com selênio, estes comumente variando conforme a área geográfica de plantio ou criação do animal Kieliszek (2019).

A disponibilidade e a mobilidade das diferentes espécies de Se disponíveis no solo determinam a quantidade de Se absorvida pelas plantas. A absorção, por sua vez, é influenciada pela complexidade das reações químicas e propriedades físicoquímicas do solo, pelo tipo de planta, o regime de gestão e as condições climáticas Dinh et al. (2018).

Um exemplo específico de condições climáticas evidenciado é o solo do cerrado brasileiro, onde as concentrações de Se, por sua vez, encontram-se abaixo do valor da qualidade do solo Carvalho, Oliveira, Curi, Schulze, Marques (2019). As raízes das castanheiras do Brasil, por sua vez, possuem concentrações de Se elevadas, sendo esta ocorrência de maneira independente da idade das castanheiras Castro et al. (2019).

Outro fator determinante para o acúmulo desse mineral, está no uso de fertilizante com Se, que favorece as culturas 
forrageiras, que se caracterizam por absorverem e acumularem mais Se quando comparadas, por exemplo, com as culturas de grãos Ebrahimi, Stoddard, Hartikainen, Seppãnen (2019).

O consumo total médio de Se pode ser estimado pelo conteúdo de Se no alimento, em que este, por sua vez, é fortemente determinado pelo solo. Sendo assim, os altos valores de Se para o solo são refletidos em altas concentrações de $\mathrm{Se}$ em animais e plantas locais Navarro-Alarcon, Cabrera-Vique (2008). Desse modo, as culturas forrageiras que servem de alimento para o gado leiteiro influenciam diretamente a composição do leite bovino Simões, da Silva, de Oliveira, Cristaldo, Brito (2009).

Sob esse prisma, um estudo anterior relacionado à composição de alimentos expôs resultados referentes à análise de selênio em mel proveniente do estado do Ceará. Percebe-se um aumento na concentração de Se nos alimentos, de 3,6 $\mu \mathrm{g} \mathrm{kg-1}$ no milho e 46,4 $\mu \mathrm{g} \mathrm{kg-1} \mathrm{no} \mathrm{feijão.} \mathrm{Sendo} \mathrm{este} \mathrm{último} \mathrm{grão} \mathrm{que} \mathrm{melhor} \mathrm{responde} \mathrm{ao} \mathrm{fertilizante} \mathrm{Ngigi,} \mathrm{Lachat,} \mathrm{Masinde,} \mathrm{Du}$ Laing (2019). Contudo, ainda existe uma lacuna na área de composição de alimentos do Ceará e do Brasil ao caracterizar a concentração de selênio em leites da região, que precisa ser preenchida, visto que as tabelas de composição de alimentos são fundamentais para pesquisadores e profissionais da área, os quais precisam de informações seguras, a fim de minimizar as possibilidades de erro nos planejamentos alimentares e pesquisas científicas.

Assim, objetivou-se determinar a concentração de selênio e a composição centesimal do leite bovino in natura originados do estado do Ceará - Brasil.

\section{Metodologia}

A pesquisa possui um caráter observacional descritivo. As amostras de leite bovino in natura foram coletadas em municípios de oito bacias leiteiras, totalizando todas as existentes no estado do Ceará, na qual fornecem leite bovino para empresas produtoras de alimentos.

Os procedimentos de armazenagem, acondicionamento e preparo das amostras foram realizados no Laboratório de Análises de Alimentos e Micronutrientes da Universidade Estadual do Ceará. Todo material utilizado foi previamente desmineralizado em banho de ácido nítrico $20 \%$.

\section{Amostragem: Leite Bovino in natura}

As amostras de leite provenientes das bacias de Fortaleza, Quixeramobim e Vale do Curu foram adquiridas de tanques de coleta de uma cooperativa agrícola cearense que recebe leite de várias regiões do estado. Nestes tanques são coletados leites de diferentes municípios de cada uma destas bacias formando um pool da produção de cada bacia. Para análise em laboratório foram coletados $900 \mathrm{~mL}$ de cada um dos tanques. As amostras de leite das bacias leiteiras do Médio Jaguaribe, Alto Salgado e Cariri foram provenientes de um único produtor por bacia e as amostras das bacias de Sobral e Crateús originaram-se de dois diferentes produtores por bacia leiteira.

No período da coleta das amostras os produtores utilizavam predominantemente forragens plantadas em seus municípios para alimentação do gado leiteiro. Tipicamente, no território da região do estado do Ceará, as forragens utilizadas envolvem as espécies catingueira (Caesalpinia bracteosa), jucazeiro (Caesalpinia ferrea), jurema preta (Mimosa hostillis) e sabiá (Mimosa caesalpinifolia) Nozella et al (2006); Antonio (2015).

\section{Preparo das Amostras}

As amostras de leite in natura foram congeladas e transportadas em recipientes térmicos para o Laboratório de Análises de Alimentos e Micronutrientes - UECE, sendo o leite previamente homogeneizado por inversão. O preparo, a conservação e a homogeneização das amostras de leite foram feitas em concordância com os métodos propostos pelo Instituto 
Adolfo Lutz Lutz (2008).

\section{Determinação da Composição Centesimal}

Os teores de umidade e cinzas das amostras foram determinados de acordo com a Association of Official Analytical Chemists AOAC (1980). Para a determinação do teor de proteínas utilizou-se o método de Micro Kjeldahl AOAC (1980); para o teor de lipídios o método de Soxhlet e a quantidade de carboidratos foi calculada pela diferença. A composição centesimal foi realizada para caracterizar as amostras estudadas.

\section{Determinação da Concentração de Selênio}

A determinação da concentração de selênio foi realizada no Laboratório de Nutrição e Minerais da Universidade de São Paulo (USP) por Espectrofotometria de Absorção Atômica por Geração de Hidretos acoplado à cela de quartzo, adaptada às normas seguidas pelo laboratório. Os resultados foram expressos em $\mu \mathrm{g} / 100 \mathrm{~g}$ de amostra (base úmida).

Todo o material utilizado foi desmineralizado. As amostras passaram por processo de digestão com $6 \mathrm{~mL}$ de ácido nítrico e $1 \mathrm{~mL}$ de ácido clorídrico, inicialmente em fluxo laminar por 24 horas e posteriormente em bloco digestor a $150^{\circ} \mathrm{C}$ por 12 horas. Após a digestão, as amostras foram reduzidas, adicionando $5 \mathrm{~mL}$ de ácido clorídrico 1,2 N nos tubos de quartzo. Este material foi despejado em balão volumétrico de $50 \mathrm{~mL}$. Lavou-se o tubo de quartzo e o funil com água nanopura por três vezes e completou-se o volume do balão com a mesma água. Após este processo de diluição, as amostras foram encaminhadas para a análise em triplicata em espectrofotômetro de modelo Z-5000 (Hitachi).

A avaliação da metodologia foi realizada com o material de referência "NIST Whole Milk Powder ${ }^{\circledR}$ " nas mesmas condições de análise do leite.

\section{Análise estatística}

Inicialmente foram determinadas a normalidade e a homogeneidade dos dados através dos testes de Shapiro-Wilk e Levene, respectivamente. Os dados foram expressos em média e desvio padrão. A comparação entre as médias nas variáveis não paramétricas foi realizada pelo teste Kruskal-Wallis, seguido de pós teste de Games-Howell ou Dunnett T3 para as comparações múltiplas. Para as variáveis paramétricas, o teste utilizado foi a ANOVA, seguida do teste DMS para as comparações múltiplas. O programa utilizado nas análises foi o Statistical Package for the Social Sciences (SPSS) versão 22.0, sendo considerado significante o valor de $\mathrm{p}<0,05$.

\section{Resultados e Discussão}

Na Tabela 1 são apresentados os resultados relativos ao teor de selênio e composição centesimal das amostras de leite bovino in natura. Percebe-se uma larga variação entre as concentrações de $\mathrm{Se}$, inclusive entre localizações com menores distâncias, tais como a capital Fortaleza até Maranguape na Região Metropolitana (22 km) e Quixadá até Quixeramobim, ambos no Sertão Central $(39 \mathrm{~km})$. Todas as variáveis apresentaram diferenças significativas entre os municípios, sendo as diferenças individuais demonstradas na Tabela 1, com destaque para as maiores concentrações de Se em Quixeramobim. 
Tabela 1: Caracterização do teor de Se e composição centesimal do leite bovino in natura, de acordo com o município da base úmida provenientes do estado do Ceará. Fortaleza, 2014.

\begin{tabular}{ccccccc}
\hline Municípios & Umidade & Cinza & Proteína & Lipídio & Carboidrato & Selênio \\
\hline Crateús & $88,87(0,77)^{\mathrm{a}}$ & $0,58(0,04)^{\mathrm{a}}$ & $2,84(0,22)$ & $2,97(0,19)^{\mathrm{a}}$ & $4,74(0,66)^{\mathrm{a}}$ & $1,1(0,02)^{\mathrm{a}}$ \\
Crato & $89,36(0,04)^{\mathrm{b}}$ & $0,68(0,00)^{\mathrm{ab}}$ & $2,94(0,07)^{\mathrm{a}}$ & $2,32(0,03)^{\mathrm{ab}}$ & $4,69(0,06)^{\mathrm{b}}$ & $2,87(0,40)^{\mathrm{b}}$ \\
Fortaleza & $88,10(0,03)^{\mathrm{bc}}$ & $0,70(0,10)$ & $2,90(0,06)$ & $4,83(0,71)$ & $3,47(0,72)^{\mathrm{abc}}$ & $0,34(0,01)^{\mathrm{abc}}$ \\
Iguatu & $87,29(0,19)^{\mathrm{abd}}$ & $0,71(0,14)$ & $3,30(0,36)$ & $4,20(0,36)^{\mathrm{c}}$ & $4,50(0,54)^{\mathrm{cd}}$ & $4,00(0,01)^{\mathrm{acd}}$ \\
Itapipoca & $89,79(1,15)^{\mathrm{d}}$ & $0,60(0,16)$ & $2,87(0,96)$ & $2,96(0,31)^{\mathrm{bcd}}$ & $3,78(0,77)^{\mathrm{abde}}$ & $1,21(0,03)^{\mathrm{acde}}$ \\
Limoeiro & $88,90(0,30)^{\mathrm{d}}$ & $0,63(0,01)^{\mathrm{c}}$ & $2,66(0,07)$ & $3,67(0,03)^{\mathrm{abde}}$ & $4,14(0,33)$ & $0,89(0,26)^{\mathrm{bd}}$ \\
Maranguape & $89,10(0,37)^{\mathrm{d}}$ & $0,39(0,25)$ & $2,63(0,06)^{\mathrm{a}}$ & $3,08(0,02)^{\mathrm{bef}}$ & $4,81(0,50)^{\mathrm{cef}}$ & $5,48(1,23)$ \\
Quixadá & $88,94(0,20)^{\mathrm{d}}$ & $0,45(0,02)^{\mathrm{abc}}$ & $2,83(0,10)$ & $3,22(0,02)^{\mathrm{befg}}$ & $4,57(0,13)^{\mathrm{c}}$ & $0,09(0,00)^{\mathrm{abcdef}}$ \\
Quixeramobim & $91,74(0,86)$ & $0,55(0,10)$ & $2,18(0,18)$ & $2,14(0,01)^{\mathrm{abcdefgh}}$ & $3,39(0,59)^{\mathrm{abdfg}}$ & $8,9(0,59)^{\mathrm{abcdefg}}$ \\
Umirim & $89,51(0,14)^{\mathrm{cd}}$ & $0,35(0,23)$ & $2,80(0,18)$ & $2,57(0,00)^{\mathrm{abefgh}}$ & $4,78(0,51)^{\mathrm{ceg}}$ & $4,09(0,04)^{\mathrm{acefg}}$ \\
Total & $89,04(1,18)$ & $0,58(0,17)$ & $2,87(0,46)$ & $3,15(0,85)$ & $4,35(0,72)$ & $2,90(2,73)$ \\
Valor p & $0,008^{¥}$ & $0,041^{\ddagger}$ & $0,042^{\mathfrak{f}}$ & $<0,001^{\ddagger}$ & $0,010^{\ddagger}$ & $0,001^{\ddagger}$ \\
\hline
\end{tabular}

Valores descritos em média (desvio padrão); ${ }^{¥}$ Teste Kruskal Wallis com pós-teste de Games-Howell; ${ }^{£}$ Teste Kruskal Wallis com pós-teste de Dunnett T3; ${ }^{A}$ ANOVA com pós-teste DMS; Letras iguais representam diferença estatística entre os grupos; Valor de $p$ considerado significativo sendo menor ou igual a 0,05 . Fonte: Autores.

Os valores proteicos encontrados nesse estudo são menores do que os apresentados por Stergiadis, Nørskov, Purup, Givens, Lee (2019), os quais observaram em média 3,27g de proteína nos leites comercialmente distribuídos no Reino Unido. Em contrapartida, valores protéicos encontrados por Bondan, Folchini, Noro, Machado, Muhls, González (2019), em amostras de leite coletadas no estado do Rio Grande do Sul (RS), foram entre 2,9 a 3,1g/100mL de leite. Ainda, de acordo com os mesmos autores, os valores de lipídios foram considerados semelhantes com o atual trabalho, onde a média encontrada em amostras de leite no Reino Unido foi de 3,49g e, no estado do RS foi de 3,39 g/100mL.

A biodisponibilidade do selênio nos solos varia de acordo com as suas características, em especial o pH e o tipo de rocha da qual foram originados Eiche, Nothstein, Gottlicher, Steininger, Dhillon, Neumann (2019). Dados disponibilizados pela Fundação Cearense de Meteorologia e Recursos Hídricos mostram que o Ceará possui uma estrutura geológica extremamente heterogênea e complexa resultante de uma variação de terrenos associados ao clima semi-árido, havendo o predomínio dos solos Neossolos (36\% do território), Argissolos $(24,7 \%)$ e Luvissolos (16,7\%), podendo ser uma justificativa plausível para as variações nas concentrações de Se entre os 10 municípios do estado.

Algumas concentrações de selênio em amostras de tipos de leites de diferentes regiões do mundo foram apresentadas no Quadro 1. 
Quadro 1: Teor de selênio em leites de diferentes regiões do mundo.

\begin{tabular}{|l|c|c|c|}
\hline \multicolumn{1}{|c|}{$\begin{array}{c}\text { Especificação } \\
\text { do leite }\end{array}$} & $\begin{array}{c}\text { Teor médio } \\
\text { de selênio }\end{array}$ & País & Referência \\
\hline Integral, past. UHT & $2.8(0.6)^{\mathrm{a}}$ & Tailândia & Sirichakwal et al. (2005) \\
\hline Integral, in natura & $2.3^{\text {ab }}$ & Holanda & Van Hulzen et al. (2009) \\
\hline Integral, in natura & $2.0(0.6) \mu \mathrm{g} / 100 \mathrm{~mL}$ & Polônia & Pilarczyk et al. (2010) \\
\hline Integral, in natura & $2.0 \mu \mathrm{g} / 100 \mathrm{~mL}$ & EUA $^{\mathrm{c}}$ & USDA $^{\mathrm{d}}(2011)$ \\
\hline Integral, in natura & $3.7 \mu \mathrm{g} / 100 \mathrm{~mL}$ & México & Brasil $^{\mathrm{e}}(2011)$ \\
\hline Integral, in natura & $4.6(0.1)^{\mathrm{a}}$ & Estônia & Ling et al. (2017) $^{\mathrm{a}}$ \\
\hline Não pasteurizado & $1.8(0.02)^{\mathrm{a}}$ & Irlanda do Norte & O’kane et al. (2018) $^{\mathrm{a}}$ \\
\hline
\end{tabular}

${ }^{\mathrm{a} C o n c e n t r a c ̧ a ̃ o ~ e x p r e s s a ~ e m ~} \mu \mathrm{g} / 100 \mathrm{~g}$ de peso úmido.

${ }^{\mathrm{b}}$ Coeficiente de variação $=0.2 \mu \mathrm{g} / 100 \mathrm{~g}$

${ }^{\mathrm{c}}$ Estados Unidos das Américas.

d United States Department of Agriculture

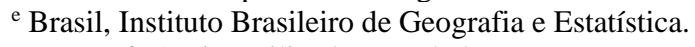

Fonte: referências utilizadas na tabela.

Ao comparar leites in natura de diferentes países com o leite in natura proveniente do estado do Ceará observa-se que há bastante variação, sendo que Quixadá, Fortaleza, Limoeiro do Norte, Crateús e Itapipoca apresentaram valores ainda menores que Irlanda do Norte, e em Maranguape e Quixeramobim, os valores foram maiores que na Estônia.

As variações nas concentrações de micronutrientes e/ou macronutrientes presentes no leite bovino podem ocorrer também devido a fatores relacionados ao clima, estação do ano, características nutricionais do alimento ingerido pelo animal, além do número de vezes em que o animal foi ordenhado, bem como o horário da ordenha Verduci et al. (2019).

Neste estudo destaca-se que as diferenças na alimentação do gado, informados pelos produtores, foram pequenas, e os três anos de coleta foram anos de seca no estado. Assim, pode-se estimar que as variações da concentração de selênio nas amostras provenientes das diferentes regiões do Ceará refletem a multiplicidade geográfica dos solos em relação ao selênio. Diante disso, e tendo em vista que a composição de selênio nos laticínios é diretamente influenciada pelo consumo da ração, uma alternativa interessante seria suplementar a ração animal com selênio a fim de garantir uma concentração mais elevada nos produtos resultantes da atividade leiteira Liu, Zhu, Lu, Wei, Ren (2015); Ling et al. (2017).

Segundo o Ministério da Saúde, a recomendação do consumo de leite e seus derivados varia de acordo com a idade das pessoas. A recomendação para crianças de até 10 anos é de $400 \mathrm{~mL} /$ dia, já para adultos acima de 20 anos, recomenda-se $600 \mathrm{~mL} /$ dia Portal Brasil (2015). Podendo assim suprir parte das necessidades de macro e micronutrientes de cada grupo, e melhorar a saúde humana e imunidade, e prevenir ou tratar ameaças à saúde, uma vez que o leite e os seus produtos têm atividades antioxidantes (Khan, Bule, Rahman Ullah, Asif, Niaz (2019).

Diante desse volume recomendado, se faz necessário o desenvolvimento de políticas públicas para orientações de consumo, bem como incentivos para a atividade econômica da pecuária leiteira Juntolli (2016). Vale ressaltar que as propriedades agropecuárias no Ceará são representadas por $43 \%$ de sua totalidade sendo de origem familiar, onde $74 \%$ apresenta rebanho de até dezenove cabeças de gado e, ainda, há o predomínio de pequenas propriedades agropecuárias, 
caracterizadas por até 500 hectares em estimados 75\% das propriedades IPECE (2018).

Uma limitação do estudo é o fato de as amostras terem sido analisadas apenas na forma in natura, podendo o cozimento influenciar na concentração e biodisponibilidade de selênio Khanam, Platel (2016). Avaliar leites que passaram por tratamento térmico seria importante, visto que esta é a forma na qual é consumido.

O estudo de Csapó et al. (2015) mostra que seria uma alternativa enriquecer o leite com selênio, para a partir daí elaborar produtos lácteos, podendo ser uma valiosa fonte de selênio tanto para pessoas quanto para animais.

A fertilização com selênio em solos tropicais baixos de Se, como áreas agrícolas do Brasil, é uma necessidade emergente para a saúde animal e humana, e também pode ser benéfica para as plantas. Assim, a fertilização com Se em pastagens é uma alternativa para colaborar com a suplementação animal e as demandas nutricionais humanas. No entanto, são necessárias mais pesquisas em ambientes tropicais para estabelecer taxas de Se, plantas e respostas de animais e reduzir ou até neutralizar os riscos de toxicidade, mesmo que os benefícios já sejam conhecidos De Abreu Faria, Cerqueira Luz, Abdalla (2019).

Uma limitação do estudo é o fato das amostras terem sido analisadas apenas na forma in natura, podendo o cozimento influenciar na concentração e biodisponibilidade de selênio Khanam, Platel (2016). Avaliar leites que passaram por tratamento térmico seria importante, visto que esta é a forma na qual é mais consumido. Embora o estudo tenha apresentado esta limitação, destaca-se que os dados de Se em alimentos cultivados, industrializados e comercializados no Brasil são ausentes em tabelas de composição de alimentos brasileiras, sendo necessário a utilização de tabelas que compilam dados de variadas fontes bibliográficas, como a do Instituto Brasileiro de Geografia e Estatística Brasil (2011), os quais são oriundos do programa de análises de dietas Nutrition Data System for Research (NDSR), do Centro Coordenador de Nutrição (NCC) da Universidade de Minnesota, versão 2008, Estados Unidos.

Assim, esses resultados preenchem uma lacuna nessa área de pesquisa ao fornecer dados das concentrações de selênio no leite bovino provenientes do estado do Ceará e no Brasil, anteriormente indisponíveis. Essas informações podem servir para outros estudos e também para serem utilizadas em bases de dados nacionais e regionais.

\section{Conclusão}

Conclui-se que o leite bovino in natura proveniente do Ceará possui grande variação na concentração de selênio, sendo em algumas localidades superior quando relacionados com outras localidades do Brasil e do mundo. Fato que pode contribuir para o consumo deste alimento pela população do estado.

\section{Referências}

Antonio, R. P. Espécies forrageiras: principais contribuições, estado atual e perspectivas para a pesquisa no Embrapa semiárido. Empresa Brasileira de Pesquisa Agropecuária (EMBRAPA), Petrolina, 2015.

Association of Official Analytical Chemists, \& Association of Official Agricultural Chemists (US). (1980). Official methods of analysis (Vol. 13).

Bondan, C., Folchini, J. A., Noro, M., Machado, K. M., Muhls, E., \& González, F. H. D. (2019). Variation of cow’s milk composition across different daily milking sessions and feasibility of using a composite sampling. Ciência Rural, 49(6).

Brasil, I. B. G. E. (2011). Instituto Brasileiro de Geografia e Estatística. Pesquisa de Orçamentos Familiares 2008-2009: Tabelas de Composição Nutricional dos Alimentos Consumidos no Brasil. Rio de Janeiro, p. 251.

Castro, D. A., Souza, J. H. R., Moraes, M. F., Wilson, L., Broadley, M. R., Tardin, A. B. B., \& White, P. J. (2019, October). Accumulation and distribution of selenium in Brazil nut tree in relation to soil selenium availability. In Selenium Research for Environment and Human Health: Perspectives, Technologies and Advancements: Proceedings of the 6th International Conference on Selenium in the Environment and Human Health (ICSEHH 2019). CRC Press, 408(22), october, 2019.

Csapó, J., Holló, G., Holló, I., Salamon, R. V., Salamon, S., Toró, S., \& Csapóné Kiss, Z. (2015). Production of selenium-enriched milk and dairy products. Acta Univ. Sapientiae Aliment, 8, 5-29. 
Carvalho, G. S., Oliveira, J. R., Curi, N., Schulze, D. G., \& Marques, J. J. (2019). Selenium and mercury in Brazilian Cerrado soils and their relationships with physical and chemical soil characteristics. Chemosphere, 218, 412-415.

De Abreu Faria, L., de Cerqueira Luz, P. H., \& Abdalla, A. L. (2019). Selenium Fertilization in Tropical Pastures. In Importance of Selenium in the Environment and Human Health. IntechOpen.

Dinh, Q. T., Wang, M., Tran, T. A. T., Zhou, F., Wang, D., Zhai, H., \& Liang, D. (2019). Bioavailability of selenium in soil-plant system and a regulatory approach. Critical Reviews in Environmental Science and Technology, 49(6), 443-517. 2019.

Ebrahimi, N., Stoddard, F. L., Hartikainen, H., \& Seppänen, M. M. (2019). Plant species and growing season weather influence the efficiency of selenium biofortification. Nutrient Cycling in Agroecosystems, 114(2), 111-124. 2019.

Eiche, E., Nothstein, A. K., Göttlicher, J., Steininger, R., Dhillon, K. S., \& Neumann, T. (2019). The behaviour of irrigation induced Se in the groundwatersoil-plant system in Punjab, India. Environmental Science: Processes \& Impacts, 21(6), 957-969. 2019.

Freitas, G. C. V., Lemos, R. D. C. A. S., França, A. C. B., dos Santos, L. C. C., Leão, S. M. L. M., \& Nogueira, T. R. (2020). Efeitos da suplementação de selênio sobre a defesa antioxidante na Doença Renal Crônica. Research, Society and Development, 9(5), e189953247-e189953247. 2020.

IPECE (2018). Análise da cadeia produtiva do leite e seus derivados no Ceará. IPECE informe / Instituto de Pesquisa e Estratégia Econômica do Ceará (IPECE) Fortaleza-Ceará: Secretaria do Planejamento e Gestão do Estado do Ceará. Informe número 128.

Juntolli, F. (2016). A pecuária de leite no Brasil: cenários e avanços tecnológicos. Brasília, DF: Embrapa.

Khanam, A., \& Platel, K. (2016). Bioaccessibility of selenium, selenomethionine and selenocysteine from foods and influence of heat processing on the same. Food chemistry, 194, 1293-1299. 2016.

Khan, I. T., Bule, M., Rahman Ullah, M. N., Asif, S., \& Niaz, K. (2019). The antioxidant components of milk and their role in processing, ripening, and storage: Functional food. Veterinary world, 12(1), 12. 2019.

Kieliszek, M., \& Błażejak, S. (2016). Current knowledge on the importance of selenium in food for living organisms: a review. Molecules, 21(5), 609.2016.

Kieliszek, M. (2019). Selenium-fascinating microelement, properties and sources in food. Molecules, 24(7), 1298. 2019.

Ling, K., Henno, M., Jõudu, I., Püssa, T., Jaakson, H., Kass, M., \& Ots, M. (2017). Selenium supplementation of diets of dairy cows to produce Se-enriched cheese. International dairy journal, 71, 76-81. 2017.

Liu, H. Y., Zhu, W. Z., Lu, B. Y., Wei, Z. H., \& Ren, D. X. (2015). Effect of feed selenium supplementation on milk selenium distribution and mozzarella quality. Journal of Dairy Science, 98(12), 8359-8367. 2015.

Lutz, I. A. (2008). Métodos físico-químicos para análise de alimentos. São Paulo: Agência Nacional de Vigilância Sanitária (ANVISA). 2018.

Navarro-Alarcon, M., \& Cabrera-Vique, C. (2008). Selenium in food and the human body: a review. Science of the total environment, 400(1-3), 115-141. 2008 .

Nozella, E. F., Cabral-Filho, S. L. S., Bueno, I. C. S., Godoy, P. B., Minho, A. P., Araújo-Filho, J. A., \& Vitti, D. M. S. S. Caracterização de forrageiras do nordeste utilizando a técnica de produção de gases, quantificação de taninos e composição química. 2. Plantas do estado do Ceará. Empresa Brasileira de Pesquisa Agropecuária (EMBRAPA), 1-4, 2006.

Ngigi, P. B., Lachat, C., Masinde, P. W., \& Du Laing, G. (2019). Agronomic biofortification of maize and beans in Kenya through selenium fertilization. Environmental geochemistry and health, 41(6), 2577-2591. 2019.

O'Kane, S. M., Pourshahidi, L. K., Mulhern, M. S., Weir, R. R., Hill, S., O'Reilly, J., \& Yeates, A. J. (2018). The effect of processing and seasonality on the iodine and selenium concentration of cow's milk produced in Northern Ireland (NI): Implications for population dietary intake. Nutrients, 10(3), 287. 2018.

Pilarczyk, B., Tomza-Marciniak, A., Mituniewicz-Małek, A., Wieczorek-Dąbrowska, M., Pilarczyk, R., Wójcik, J., \& Dmytrów, I. (2010). Selenium content in selected products of animal origin and estimation of the degree of cover daily Se requirement in Poland. International journal of food science \& technology, 45(1), 186-191. 2010

Portal Brasil (2015, abril 2). Ministério da Agricultura quer fomentar o consumo de leite. http://www.brasil.gov.br/economia-e-emprego/2015/03/ministerioda-agricultura-quer-fomentar-o-consumo-de-leite

Rostami, S., Fathollahpour, A., Abdi, M., \& Naderi, K. (2018). Alteration in prooxidant-antioxidant balance associated with selenium concentration in patients with congenital hypothyroidism. Journal of medical biochemistry, 37(3), 355. 2018.

Simões, A. R. P., da Silva, R. M., de Oliveira, M. V. M., Cristaldo, R. O., \& Brito, M. C. B. (2009). Economic evaluation of three different milk production systems in the Alto Pantanal Sul-mato-grossense. Revista Agrarian, 2(5), 153-167. 2009.

Sirichakwal, P. P., Puwastien, P., Polngam, J., \& Kongkachuichai, R. (2005). Selenium content of Thai foods. Journal of Food Composition and Analysis, 18(1), 47-59. 2005.

Stergiadis, S., Nørskov, N. P., Purup, S., Givens, I., \& Lee, M. R. (2019). Comparative nutrient profiling of retail goat and cow milk. Nutrients, 11(10), 2282. 2019.

Tan, H. W., Mo, H. Y., Lau, A. T., \& Xu, Y. M. (2019). Selenium species: current status and potentials in cancer prevention and therapy. International journal of molecular sciences, 20(1), 75. 2019.

USDA (2013) Agricultural Research Service USDA National Nutrient Database for Standard Reference, Release 26. 
Research, Society and Development, v. 10, n. 6, e48010616011, 2021

(CC BY 4.0) | ISSN 2525-3409 | DOI: http://dx.doi.org/10.33448/rsd-v10i6.16011

Valea, A., \& Georgescu, C. E. (2018). Selenoproteins in human body: focus on thyroid pathophysiology. Hormones, 17(2), 183-196. 2018.

Van Hulzen, K. J. E., Sprong, R. C., Van der Meer, R., \& Van Arendonk, J. A. M. (2009). Genetic and nongenetic variation in concentration of selenium, calcium, potassium, zinc, magnesium, and phosphorus in milk of Dutch Holstein-Friesian cows. Journal of Dairy Science, 92(11), 5754-5759. 2009.

Verduci, E., D’Elios, S., Cerrato, L., Comberiati, P., Calvani, M., Palazzo, S., \& Peroni, D. G. (2019). Cow’s milk substitutes for children: Nutritional aspects of milk from different mammalian species, special formula and plant-based beverages. Nutrients, 11(8), 1739. 2019.

Wang, N., Tan, H. Y., Li, S., Xu, Y., Guo, W., \& Feng, Y. (2017). Supplementation of Micronutrient Selenium in Metabolic Diseases: Its Role as an Antioxidant. Oxidative medicine and cellular longevity, 2017, 7478523. https://doi.org/10.1155/2017/7478523

Zhang, Q., Li, W., Wang, J., Hu, B., Yun, H., Guo, R., \& Wang, L. (2019). Selenium levels in community dwellers with type 2 diabetes mellitus. Biological trace element research, 191(2), 354-362. 2019. 